\title{
Jacobian Consistency of a Smoothing Function for the Weighted Second-Order Cone Complementarity Problem
}

\author{
Wenli Liu, ${ }^{1}$ Xiaoni Chi $\mathbb{D}^{2}{ }^{2}$ Qili Yang, ${ }^{1}$ and Ranran Cui ${ }^{1}$ \\ ${ }^{1}$ School of Mathematics and Computing Science, Guangxi Key Laboratory of Cryptography and Information Security, \\ Guilin University of Electronic Technology, Guilin 541004, China \\ ${ }^{2}$ School of Mathematics and Computing Science, Guangxi Key Laboratory of Automatic Detecting Technology and Instruments, \\ Guilin University of Electronic Technology, Guilin 541004, China \\ Correspondence should be addressed to Xiaoni Chi; chixiaoni@126.com
}

Received 12 November 2020; Revised 14 December 2020; Accepted 4 January 2021; Published 23 January 2021

Academic Editor: Guoqiang Wang

Copyright (@) 2021 Wenli Liu et al. This is an open access article distributed under the Creative Commons Attribution License, which permits unrestricted use, distribution, and reproduction in any medium, provided the original work is properly cited.

In this paper, a weighted second-order cone (SOC) complementarity function and its smoothing function are presented. Then, we derive the computable formula for the Jacobian of the smoothing function and show its Jacobian consistency. Also, we estimate the distance between the subgradient of the weighted SOC complementarity function and the gradient of its smoothing function. These results will be critical to achieve the rapid convergence of smoothing methods for weighted SOC complementarity problems.

\section{Introduction}

The weighted second-order cone complementarity problem (WSOCCP) is, for a given weight vector $w \in \mathscr{K}$ and a continuously differentiable function $F: \mathbb{R}^{n} \times \mathbb{R}^{n} \times \mathbb{R}^{m} \longrightarrow$ $\mathbb{R}^{n+m}$, to find vectors $(x, s, y) \in \mathbb{R}^{n} \times \mathbb{R}^{n} \times \mathbb{R}^{m}$ such that

$$
\left\{\begin{array}{l}
x \circ s=w, \\
F(x, s, y)=0, \\
x \in \mathscr{K}, \\
s \in \mathscr{K},
\end{array}\right.
$$

where $\circ$ represents the Jordan product and $\mathscr{K}$ is the Cartesian product of second-order cone, that is, $\mathscr{K}=\mathscr{K}^{n_{1}} \times$ $\mathscr{K}^{n_{2}} \times \cdots \times \mathscr{K}^{n_{r}}$ with $\sum_{i=1}^{r} n_{i}=n, i=1, \ldots, r$. The set $\mathscr{K}^{n_{i}}(i=1, \ldots, r)$ is the second-order cone (SOC) of dimension $n_{i}$ defined by

$$
\mathscr{K}^{n_{i}}:=\left\{x_{i}=\left(x_{i 0}, x_{i 1}\right) \in \mathbb{R} \times \mathbb{R}^{n_{i}-1}: x_{i 0}-\left\|x_{i 1}\right\| \geq 0\right\},
$$

and the interior of the SOC $\mathscr{K}^{n_{i}}$ is the set

$$
\operatorname{int} \mathscr{K}^{n_{i}}=\left\{x_{i}=\left(x_{i 0}, x_{i 1}\right) \in \mathbb{R} \times \mathbb{R}^{n_{i}-1}: x_{i 0}-\left\|x_{i 1}\right\|>0\right\} .
$$

Here $\|\cdot\|$ is the Euclidean norm, and

$$
\operatorname{int} \mathscr{K}=\operatorname{int} \mathscr{K}^{n_{1}} \times \operatorname{int} \mathscr{K}^{n_{2}} \times \cdots \times \operatorname{int} \mathscr{K}^{n_{r}} .
$$

Obviously, if $w=0$, WSOCCP (1) reduces to second-order cone complementarity problem (SOCCP). In this article, we may assume that $r=1$ and $\mathscr{K}=\mathscr{K}^{n}$ in the following analysis, since it can easily be extended to the general case.

In order to reformulate several equilibrium problems in economics and study highly efficient algorithms to solve these problems, Potra [1] introduced the notion of a weighted complementarity problem (WCP). He showed that the Fisher market equilibrium problem can be modeled as a monotone linear WCP. Moreover, the linear programming and weighted centering (LPWC) problem, which was introduced by Anstreicher [2], can also be formulated as a monotone linear WCP. And Potra [1] analyzed two interiorpoint methods for solving the monotone linear WCP over the nonnegative orthant. Since then, many scholars are dedicated to investigating the theories and solution methods 
of WCP. Tang [3] gave a new nonmonotone smoothing-type algorithm to solve the linear WCP. Chi et al. [4] studied the existence and uniqueness of the solution for a class of WCPs.

As is well known, smoothing methods have superior theoretical and numerical performances. For solving the SOCCP by smoothing methods, we usually reformulate the SOCCP as a system of equations based on parametric smoothing functions of SOC complementarity functions $[5,6]$. The smoothing parameter involved in smoothing functions may be treated as a variable [7] or a parameter with an appropriate parameter control [8]. In the latter case, the Jacobian consistency is important to achieve a rapid convergence of Newton methods or Newton-like methods. Hayashi et al. [8] proposed a combined smoothing and regularized method for monotone SOCCP, and based on the Jacobian consistency of the smoothing natural residual function, they proved that the method has global and quadratic convergence. Krejić and Rapajić [9] gave a nonmonotone Jacobian smoothing inexact Newton method for nonlinear complementarity problem and proved the global and local superlinear convergence of the method. Chen et al. [10] presented a modified Jacobian smoothing method for the nonsmooth complementarity problem and established the global and fast local convergence for the method.

In this paper, we consider the function $\varphi: \mathbb{R}^{n} \times \mathbb{R}^{n} \longrightarrow$ $\mathbb{R}^{n}$ for WSOCCP

$$
\varphi(x, s, w):=x+s-\sqrt{x^{2}+s^{2}+x \circ s+w},
$$

with a given vector $w \in \mathscr{K}^{n}$. If $w=0, \varphi(5)$ reduces to the SOC complementarity function [6] with $\tau=3$ :

$$
\varphi(x, s, 0):=x+s-\sqrt{x^{2}+s^{2}+x \circ s} .
$$

Since $\varphi$ is nonsmooth, we define the following smoothing function $\varphi_{\mu}$ :

$$
\varphi_{\mu}(x, s, w):=x+s-\sqrt{x^{2}+s^{2}+x \circ s+w+\mu^{2} e},
$$

where $\mu \in \mathbb{R}$ is a smoothing parameter.

The main contribution of this paper is to show the Jacobian consistency of the smoothing function (7) and estimate the distance between the subgradient of the weighted SOC complementarity function (5) and the gradient of its smoothing function (7). These properties will be critical to solve weighted SOC complementarity problems by smoothing methods.

The paper is organized as follows. In Section 2, we review some concepts and properties. In Section 3, we derive the computable formula for the Jacobian of the smoothing function in WSOCCP. In Section 4, we show the Jacobian consistency of the smoothing function and estimate the distance between the gradient of smoothing function and the subgradient of the weighted SOC complementarity function. Some conclusions are reported in Section 5.

Throughout this paper, $\mathbb{R}_{+}$denotes the set of nonnegative numbers. $\mathbb{R}^{n}$ and $\mathbb{R}^{m \times n}$ denote the space of $n$-dimensional real column vectors and the space of matrices, respectively. We use $\|\cdot\|$ to denote the Euclidean norm and define $\|x\|:=\sqrt{x^{T} x}$ for a vector $x$ or the corresponding induced matrix norm. For simplicity, we often use $x=\left(x_{0} ; x_{1}\right)$ instead of the column vector $x=\left(x_{0}, x_{1}^{T}\right)^{T}$. $\operatorname{int} \mathscr{K}^{n}$ and bd $\mathscr{K}^{n}$ mean the topological interior and the boundary of the SOC $\mathscr{K}^{n}$, respectively. For a given set $S \subset \mathbb{R}^{m \times n}$, convS denotes the convex hull of $S$ in $\mathbb{R}^{m \times n}$, and for any matrix $X \in \mathbb{R}^{m \times n}$, $\operatorname{dist}(X, S)$ denotes $\inf \{\|X-Y\|$ : $Y \in S\}$.

\section{Preliminaries}

In this section, we briefly recall some definitions and results about the Euclidean Jordan algebra [11] associated with the SOC $\mathscr{K}^{n}$ and subdifferentials [12].

For any $x, s \in \mathbb{R}^{n}$, their Jordan product is defined as $x \circ s=\left(x^{T} s ; x_{0} s_{1}+s_{0} x_{1}\right)$, and $e=(1,0, \ldots, 0) \in \mathbb{R}^{n}$ is unit element of this algebra. Given an element $x=\left(x_{0} ; x_{1}\right) \in$ $\mathbb{R} \times \mathbb{R}^{n-1}$, we define the symmetric matrix

$$
L(x)=\left(\begin{array}{cc}
x_{0} & x_{1}^{T} \\
x_{1} & x_{0} I
\end{array}\right)
$$

where $I$ represents the $(n-1) \times(n-1)$ identity matrix. It is easy to verify that $x \circ s=L(x) s$ for any $s \in \mathbb{R}^{n}$. Moreover, $L(x)$ is positive definite (and hence invertible) if and only if $x \in \operatorname{int} \mathscr{K}^{n}$.

For each $x=\left(x_{0} ; x_{1}\right) \in \mathbb{R} \times \mathbb{R}^{n-1}$, let $\lambda_{1}, \lambda_{2}$ and $u^{(1)}, u^{(2)}$ be the spectral values and the associated spectral vectors of $x$, given by

$$
\begin{aligned}
\lambda_{i} & =x_{0}+(-1)^{i}\left\|x_{1}\right\|, \\
u^{(i)} & = \begin{cases}\frac{1}{2}\left(1 ;(-1) \frac{x_{1}}{\left\|x_{1}\right\|}\right), & \text { if } x_{1} \neq 0, \\
\frac{1}{2}\left(1 ;(-1)^{i} \bar{x}_{1}\right), & \text { otherwise, }\end{cases}
\end{aligned}
$$

for $i=1,2$, with any $\bar{x}_{1} \in \mathbb{R}^{n-1}$ such that $\left\|\bar{x}_{1}\right\|=1$. Then, $x$ admits a spectral factorization associated with SOC $\mathscr{K}^{n}$ in the form of

$$
x=\lambda_{1} u^{(1)}+\lambda_{2} u^{(2)} .
$$

For any $x=\left(x_{0} ; x_{1}\right) \in \mathbb{R} \times \mathbb{R}^{n-1}$, let $x^{\prime}=\left(x_{0} ;-x_{1}\right)[13]$. Then, $x^{\prime \prime}=x,(x+s)^{\prime}=x^{\prime}+s^{\prime}$, and $(c x)^{\prime}=c x^{\prime}$ for any $c \in \mathbb{R}$. Moreover, $x^{\circ} \mathrm{x}^{\prime}=\mathrm{x}_{0}^{2}-\left\|\mathrm{x}_{1}\right\|^{2}=0$ if $x \in \operatorname{bd} \mathscr{K}^{n}$.

Suppose that $G: \mathbb{R}^{m} \longrightarrow \mathbb{R}^{n}$ is a locally Lipschitzian function; then, from Rademacher's theorem [14], $G$ is differentiable almost everywhere. The Bouligand (B-) subdifferential and the Clarke subdifferential of $G$ at $z$ are defined by

$$
\begin{aligned}
\partial_{B} G(z) & :=\left\{\lim _{\widehat{z} \longrightarrow z} G^{\prime}(\widehat{z}): \widehat{z} \in D_{G}\right\} \text { and } \partial G(z) \\
& =\operatorname{conv}_{B} G(z),
\end{aligned}
$$

where $D_{G}$ denotes the set of points at which $G$ is differentiable. Obviously, $\partial G(z)=\left\{G^{\prime}(z)\right\}$ if $G$ is continuously differentiable at $z$. 
Definition 1 (see [12]). Let $G: \mathbb{R}^{m} \longrightarrow \mathbb{R}^{n}$ be a locally Lipschitzian function and $G_{\mu}: \mathbb{R}^{m} \longrightarrow \mathbb{R}^{n}$ be a continuously differentiable function for any $\mu>0$, and for any $z \in \mathbb{R}^{m}$, we have $\lim _{\mu \longrightarrow 0} G_{\mu}(z)=G(z)$. Then, $G_{\mu}$ satisfies the Jacobian consistency property if for any $z \in \mathbb{R}^{m}, \lim _{\mu \longrightarrow 0}$ dist $\left(G_{\mu}^{\prime}(z), \partial G(z)\right)=0$.

\section{Smoothing Function}

In this section, we study the properties of the smoothing function (7).

Definition 2 (see [8]). For a nondifferentiable function $f: \mathbb{R}^{m} \longrightarrow \mathbb{R}^{n}$, we consider a function $f_{\mu}: \mathbb{R}^{m} \longrightarrow \mathbb{R}^{n}$ with a parameter $\mu>0$ that has the following properties:

(i) $f_{\mu}$ is differentiable for any $\mu>0$

(ii) $\lim _{\mu \longrightarrow 0} f_{\mu}(x)=f(x)$ for any $x \in \mathbb{R}^{m}$

Such a function $f_{\mu}$ is called a smoothing function of $f$.

Lemma 1. For any $w \in \mathscr{K}^{n}$ and $\mu \in \mathbb{R}$, one has

$$
\varphi_{\mu}(x, s, w)=0 \Leftrightarrow x \circ s=w+\mu^{2} e, \quad x \in \mathscr{K}^{n}, s \in \mathscr{K}^{n} .
$$

Proof. We first suppose that $x \circ s=w+\mu^{2} e, x \in \mathscr{K}^{n}, s \in \mathscr{K}^{n}$. Then,

$$
\begin{aligned}
0 & =x \circ s-w-\mu^{2} e \\
& =(x+s)^{2}-\left(x^{2}+s^{2}+x \circ s+w+\mu^{2} e\right),
\end{aligned}
$$

and hence

$$
x+s=\sqrt{x^{2}+s^{2}+x \circ s+w+\mu^{2} e} .
$$

That is, $\varphi_{\mu}(x, s, w)=0$.

Conversely, suppose that $\varphi_{\mu}(x, s, w)=0$; then, it follows from (7) that

$$
x+s=\sqrt{x^{2}+s^{2}+x \circ s+w+\mu^{2} e} \in \mathscr{K}^{n} .
$$

Upon squaring both sides of it, we obtain

$$
x \circ s=w+\mu^{2} e \in \mathscr{K}^{n} .
$$

Let

$$
\omega:=x+s=\sqrt{x^{2}+s^{2}+x \circ s+w+\mu^{2} e} \in \mathscr{K}^{n},
$$

which implies

$$
\begin{aligned}
\omega & \in \mathscr{K}^{n}, \\
\omega^{2}=x^{2}+s^{2}+x \circ s+w+\mu^{2} e & \in \mathscr{K}^{n} .
\end{aligned}
$$

Therefore,

$$
\begin{aligned}
& \omega^{2}-s^{2}=x^{2}+x \circ s+w+\mu^{2} e \in \mathscr{K}^{n}, \\
& \omega^{2}-x^{2}=s^{2}+x \circ s+w+\mu^{2} e \in \mathscr{K}^{n} .
\end{aligned}
$$

Further, it follows from Proposition 3.4 [15] that

$$
\begin{gathered}
x=\omega-s \in \mathscr{K}^{n}, \\
s=\omega-x \in \mathscr{K}^{n} .
\end{gathered}
$$

Let $w=\left(w_{0} ; w_{1}\right) \in \mathscr{K}^{n}, \mu \in \mathbb{R}, x=\left(x_{0} ; x_{1}\right), s=\left(s_{0} ; s_{1}\right) \epsilon$ $\mathbb{R} \times \mathbb{R}^{n-1}$, and the mapping $v^{\mu}: \mathbb{R}^{2 n} \longrightarrow \mathbb{R} \times \mathbb{R}^{n-1}$ be defined by

$$
v^{\mu}=\left(v_{0}^{\mu} ; v_{1}^{\mu}\right)=v^{\mu}(x, s, w):=x^{2}+s^{2}+x \circ s+w+\mu^{2} e,
$$

For simplicity, we use $v$ to denote $v^{\mu}$ when $\mu=0$, that is,

$$
v=\left(v_{0} ; v_{1}\right)=v(x, s, w):=x^{2}+s^{2}+x \circ s+w \text {. }
$$

By direct calculations, we have

$$
\begin{aligned}
& v_{0}^{\mu}=\|x\|^{2}+\|s\|^{2}+x^{T} s+w_{0}+\mu^{2}=v_{0}+\mu^{2}, \\
& v_{1}^{\mu}=2 x_{0} x_{1}+2 s_{0} s_{1}+x_{0} s_{1}+s_{0} x_{1}+w_{1}=v_{1} .
\end{aligned}
$$

Therefore, $v^{\mu}=\left(v_{0}^{\mu} ; v_{1}\right)$. From the definition of spectral factorization, $v^{\mu}$ can be decomposed as

$$
v^{\mu}=\lambda_{1}\left(v^{\mu}\right) u_{1}(v)+\lambda_{2}\left(v^{\mu}\right) u_{2}(v),
$$

where $\lambda_{1}\left(v^{\mu}\right), \lambda_{2}\left(v^{\mu}\right)$, and $u_{1}(v), u_{2}(v)$ are the spectral values and the associated spectral vectors of $v^{\mu}$ given by

$$
\begin{aligned}
\lambda_{i}\left(v^{\mu}\right)= & \|x\|^{2}+\|s\|^{2}+x^{T} s+w_{0}+\mu^{2} \\
& +(-1)^{i}\left\|2 x_{0} x_{1}+2 s_{0} s_{1}+x_{0} s_{1}+s_{0} x_{1}+w_{1}\right\|,
\end{aligned}
$$

and

$$
u_{i}(v)=\frac{1}{2}\left(1 ;(-1)^{i} \bar{v}_{1}\right)
$$

for $i=1,2$, where

$$
\bar{v}_{1}:=\frac{v_{1}}{\left\|v_{1}\right\|}=\frac{2 x_{0} x_{1}+2 s_{0} s_{1}+x_{0} s_{1}+s_{0} x_{1}+w_{1}}{\left\|2 x_{0} x_{1}+2 s_{0} s_{1}+x_{0} s_{1}+s_{0} x_{1}+w_{1}\right\|},
$$

if $v_{1} \neq 0$; otherwise, $\bar{v}_{1}$ is any vector in $\mathbb{R}^{n-1}$ such that $\left\|\bar{v}_{1}\right\|=1$. For any given $w=\left(w_{0} ; w_{1}\right) \in \mathscr{K}^{n}$ and any $(x, s) \in \mathbb{R}^{n} \times \mathbb{R}^{n}$, it can be verified that

$$
\begin{aligned}
v^{\mu} & =x^{2}+s^{2}+x \circ s+w+\mu^{2} e \\
& =\left(x+\frac{s}{2}\right)^{2}+\frac{3}{4} s^{2}+w+\mu^{2} e \\
& =\left(s+\frac{x}{2}\right)^{2}+\frac{3}{4} x^{2}+w+\mu^{2} e \in \operatorname{int} \mathscr{K}^{n},
\end{aligned}
$$

for any $\mu>0$, and

$$
\begin{aligned}
v & =x^{2}+s^{2}+x \circ s+w \\
& =\left(x+\frac{s}{2}\right)^{2}+\frac{3}{4} s^{2}+w \\
& =\left(s+\frac{x}{2}\right)^{2}+\frac{3}{4} x^{2}+w \in \mathscr{K}^{n} .
\end{aligned}
$$


Given $\mu \in \mathbb{R}$ and $x=\left(x_{0} ; x_{1}\right), s=\left(s_{0} ; s_{1}\right) \in \mathbb{R} \times \mathbb{R}^{n-1}$, we define

$$
\omega^{\mu}=\left(\omega_{0}^{\mu} ; \omega_{1}^{\mu}\right)=\omega^{\mu}(x, s, w):=\sqrt{x^{2}+s^{2}+x \circ s+w+\mu^{2} e},
$$

and when $\mu=0$,

$$
\omega=\left(\omega_{0} ; \omega_{1}\right)=\omega(x, s, w):=\sqrt{x^{2}+s^{2}+x \circ s+w} .
$$

The spectral factorization of $\omega^{\mu}$ and $\omega$ is as follows:

$$
\begin{gathered}
\omega^{\mu}=\sqrt{\lambda_{1}\left(v^{\mu}\right)} u_{1}(v)+\sqrt{\lambda_{2}\left(v^{\mu}\right)} u_{2}(v), \\
\omega=\sqrt{\lambda_{1}(v)} u_{1}(v)+\sqrt{\lambda_{2}(v)} u_{2}(v) .
\end{gathered}
$$

By (29), we can partition $\mathbb{R}^{2 n}$ as $\mathbb{R}^{2 n}=\mathscr{O} \cup \mathscr{I} \cup \mathscr{B}$, where

$$
\begin{aligned}
\mathcal{O} & :=\left\{(x, s) \in \mathbb{R}^{2 n}: v \in\{0\}\right\} \\
& =\left\{(x, s) \in \mathbb{R}^{2 n}: \lambda_{2}(v)=\lambda_{1}(v)=0\right\}, \\
\mathscr{I} & :=\left\{(x, s) \in \mathbb{R}^{2 n}: v \in \operatorname{int} \mathscr{K}^{n}\right\} \\
& =\left\{(x, s) \in \mathbb{R}^{2 n}: \lambda_{2}(v) \geq \lambda_{1}(v)>0\right\}, \\
\mathscr{B} & :=\left\{(x, s) \in \mathbb{R}^{2 n}: v \in b d \mathscr{K}^{n} /\{0\}\right\} \\
& =\left\{(x, s) \in \mathbb{R}^{2 n}: 2 v_{0}=\lambda_{2}(v)>\lambda_{1}(v)=0\right\} .
\end{aligned}
$$

Lemma 2. For any given $w \in \mathscr{K}^{n}$ and any $(\mu, x, s) \in$ $\mathbb{R} \times \mathbb{R}^{n} \times \mathbb{R}^{n}$, let $\varphi$ and $\varphi_{\mu}$ be defined as (5) and (7), respectively. Then, we have

(i) The function $\varphi_{\mu}$ is continuously differentiable everywhere with any $\mu>0$, and its Jacobian is given by

$$
\varphi_{\mu}^{\prime}(x, s, w)=\left(\begin{array}{c}
I-L\left(x+\frac{s}{2}\right) L^{-1}\left(\omega^{\mu}\right) \\
I-L\left(s+\frac{x}{2}\right) L^{-1}\left(\omega^{\mu}\right)
\end{array}\right) .
$$

Here $L^{-1}\left(\omega^{\mu}\right)=\left(1 / \sqrt{v_{0}^{\mu}}\right) I$ if $v_{1}=0$; otherwise,

$$
\begin{aligned}
L^{-1}\left(\omega^{\mu}\right) & =L_{1}\left(v^{\mu}\right)+L_{2}\left(v^{\mu}\right) \\
& =\left(\begin{array}{cc}
b_{\mu} & c_{\mu} \bar{v}_{1}^{T} \\
c_{\mu} \bar{v}_{1} & a_{\mu} I+\left(b_{\mu}-a_{\mu}\right) \bar{v}_{1} \bar{v}_{1}^{T}
\end{array}\right),
\end{aligned}
$$

with

$$
\begin{aligned}
& L_{1}\left(v^{\mu}\right)=\frac{1}{2 \sqrt{\lambda_{1}\left(v^{\mu}\right)}}\left(\begin{array}{cc}
1 & -\bar{v}_{1}^{T} \\
-\bar{v}_{1} & \bar{v}_{1} \bar{v}_{1}^{T}
\end{array}\right), \\
& L_{2}\left(v^{\mu}\right)=\frac{1}{2 \sqrt{\lambda_{2}\left(v^{\mu}\right)}}\left(\begin{array}{cc}
1 & \bar{v}_{1}^{T} \\
\bar{v}_{1} & \bar{v}_{1} \bar{v}_{1}^{T}
\end{array}\right)+a_{\mu}\left(\begin{array}{cc}
0 & 0^{T} \\
0 & I-\bar{v}_{1} \bar{v}_{1}^{T}
\end{array}\right),
\end{aligned}
$$

where

$$
a_{\mu}=\frac{2}{\sqrt{\lambda_{1}\left(v^{\mu}\right)}+\sqrt{\lambda_{2}\left(v^{\mu}\right)}}
$$

$$
\begin{aligned}
& b_{\mu}=\frac{1}{2}\left(\frac{1}{\sqrt{\lambda_{1}\left(v^{\mu}\right)}}+\frac{1}{\sqrt{\lambda_{2}\left(v^{\mu}\right)}}\right), \\
& c_{\mu}=\frac{1}{2}\left(\frac{1}{\sqrt{\lambda_{2}\left(v^{\mu}\right)}}-\frac{1}{\sqrt{\lambda_{1}\left(v^{\mu}\right)}}\right) .
\end{aligned}
$$

(ii) For any $(x, s) \in \mathbb{R}^{n} \times \mathbb{R}^{n}$, we have $\lim _{\mu \longrightarrow 0} \varphi_{\mu}(x, s$, $w)=\varphi(x, s, w)$. Thus, $\varphi_{\mu}$ is a smoothing function of $\varphi$.

(iii) For any $\mu, \nu \in \mathbb{R}_{+}$,

$$
\left\|\varphi_{\mu}(x, s, w)-\varphi_{\nu}(x, s, w)\right\| \leq \sqrt{r}|\mu-\nu| .
$$

Proof

(i) For any $(x, s) \in \mathbb{R}^{n} \times \mathbb{R}^{n}$ and any $\mu>0$, according to Corollary 5.4 [15] and (28), formula (34) holds. By Proposition 5.2 and its proof [15], we get formula (35).

(ii) Given any $x=\left(x_{0} ; x_{1}\right), s=\left(s_{0} ; s_{1}\right) \in \mathbb{R} \times \mathbb{R}^{n-1}$. For any $\mu>0$, we obtain from the spectral factorization of $v^{\mu}$ and $v$ that

$$
\begin{gathered}
\varphi_{\mu}(x, s, w)=x+s-\left(\sqrt{\lambda_{1}\left(v^{\mu}\right)} u_{1}(v)+\sqrt{\lambda_{2}\left(v^{\mu}\right)} u_{2}(v)\right), \\
\varphi(x, s, w)=x+s-\left(\sqrt{\lambda_{1}(v)} u_{1}(v)+\sqrt{\lambda_{2}(v)} u_{2}(v)\right),
\end{gathered}
$$

where

$$
\begin{aligned}
\lambda_{i}(v)= & \|x\|^{2}+\|s\|^{2}+x^{T} s+w_{0}+(-1)^{i} \| 2 x_{0} x_{1}+2 s_{0} s_{1} \\
& +x_{0} s_{1}+s_{0} x_{1}+w_{1} \|
\end{aligned}
$$

and $\lambda_{i}\left(v^{\mu}\right)$ and $u_{i}(v)$ are, respectively, given by (25) and (26) for $i=1,2$. It is obvious that

$$
\lambda_{i}\left(v^{\mu}\right)=\lambda_{i}(v)+\mu^{2},
$$

for $i=1,2$. Then,

$$
\begin{aligned}
& \lim _{\mu \longrightarrow 0}\left(\sqrt{\lambda_{1}\left(v^{\mu}\right)} u_{1}(v)+\sqrt{\lambda_{2}\left(v^{\mu}\right)} u_{2}(v)\right) \\
= & \lim _{\mu \longrightarrow 0}\left(\sqrt{\lambda_{1}(v)+\mu^{2}} u_{1}(v)+\sqrt{\lambda_{2}(v)+\mu^{2}} u_{2}(v)\right) \\
= & \sqrt{\lambda_{1}(v)} u_{1}(v)+\sqrt{\lambda_{2}(v)} u_{2}(v),
\end{aligned}
$$


and $\lim _{\mu \longrightarrow 0} \varphi_{\mu}(x, s, w)=\varphi(x, s, w)$. Thus, by (i) and Definition 2, $\varphi_{\mu}$ is a smoothing function of $\varphi$.

(iii) By following the proof of Proposition 5.1 [15], we obtain the desired result.

Next, we study some properties of $\varphi$, which will be used in the subsequent analysis.

Lemma 3. For any $x=\left(x_{0} ; x_{1}\right), s=\left(s_{0} ; s_{1}\right), \quad \widetilde{w}=\left(\widetilde{w}_{0}\right.$; $\left.\widetilde{w}_{1}\right) \in \mathbb{R} \times \mathbb{R}^{n-1}$, let $x^{2}+s^{2}+\widetilde{w}^{2} \in b d \mathscr{K}^{n}$. Then, we have

$$
\begin{aligned}
x_{0}^{2} & =\left\|x_{1}\right\|^{2}, \\
s_{0}^{2} & =\left\|s_{1}\right\|^{2}, \\
\widetilde{w}_{0}^{2} & =\left\|\widetilde{w}_{1}\right\|^{2}, \\
x_{0} s_{0} & =x_{1}^{T} s_{1}, \\
x_{0} \widetilde{w}_{0} & =x_{1}^{T} \widetilde{w}_{1}, \\
s_{0} \widetilde{w}_{0} & =s_{1}^{T} \widetilde{w}_{1}, \\
x_{0} s_{1} & =s_{0} x_{1}, \\
x_{0} \widetilde{w}_{1} & =\widetilde{w}_{0} x_{1}, \\
s_{0} \widetilde{w}_{1} & =\widetilde{w}_{0}^{T} s_{1} .
\end{aligned}
$$

Proof. We can obtain the desired result by following the proof of Lemma 2 [16].

Lemma 4. For any $x=\left(x_{0} ; x_{1}\right), s=\left(s_{0} ; s_{1}\right) \in \mathbb{R} \times \mathbb{R}^{n-1}$, let $v=\left(v_{0} ; v_{1}\right)=x^{2}+s^{2}+x \circ s+w \in b d \mathscr{K}^{n}$. Then, one has

$$
\begin{aligned}
x \circ x^{\prime} & =0, \\
s \circ s^{\prime} & =0, \\
x \circ s^{\prime} & =0, \\
x \circ \widetilde{w} \prime & =0, \\
s \circ \widetilde{w} \prime & =0, \\
\widetilde{w} \circ \widetilde{w} \prime & =0, \\
x \circ v \prime & =0, \\
s \circ v \prime & =0, \\
w_{0} & =\left\|x_{0} x_{1}+s_{0} s_{1}+x_{0} s_{1}+\frac{w_{1}}{2}\right\| \\
2 & =\left\|x_{1}\right\|^{2}+\left\|s_{1}\right\|^{2}+x_{1}^{T} s_{1}+\frac{w_{1}}{2},
\end{aligned}
$$
holds:

where $\widetilde{w}:=\sqrt{w}$. Moreover, the following equivalence

$$
\begin{aligned}
v_{0} & =0 \Leftrightarrow v_{1}=0 \Leftrightarrow v=0 \\
\Leftrightarrow x_{0} & =s_{0}=w_{0}=0 \Leftrightarrow x_{1}=s_{1}=w_{1}=0 \Leftrightarrow(x, s, w)=(0,0,0) .
\end{aligned}
$$

Proof. Since

$$
\begin{aligned}
v & =x^{2}+s^{2}+x \circ s+w \\
& =\left(x+\frac{s}{2}\right)^{2}+\frac{3}{4} s^{2}+\widetilde{w}^{2} \\
& =\left(s+\frac{x}{2}\right)^{2}+\frac{3}{4} x^{2}+\widetilde{w}^{2} \in \operatorname{bd} \mathscr{K}^{n},
\end{aligned}
$$

from Lemma 3, we have

$$
\begin{aligned}
& \left(x+\frac{s}{2}\right) \circ\left(x+\frac{s}{2}\right)^{\prime}=0, \quad s \circ s^{\prime}=0, \\
& \left(x+\frac{s}{2}\right) \circ s^{\prime}=0, \quad s \circ \widetilde{w}^{\prime}=0, \\
& \left(x+\frac{s}{2}\right) \circ \widetilde{w}^{\prime}=0, \quad \widetilde{w} \circ \widetilde{w}^{\prime}=0, \\
& \left(s+\frac{x}{2} x\right) \circ\left(s+\frac{x}{2}\right)^{\prime}=0, \quad x \circ x^{\prime}=0, \\
& \left(s+\frac{x}{2}\right) \circ x^{\prime}=0, \quad x \circ \widetilde{w}^{\prime}=0, \\
& \left(s+\frac{x}{2}\right) \circ \widetilde{w}^{\prime}=0 .
\end{aligned}
$$

It follows from these equalities that the results in (45)-(47) hold. Since $v \in \operatorname{bd} \mathscr{K}^{n}$, we have $\lambda_{1}(v)=0$, i.e.,

$$
\|x\|^{2}+\|s\|^{2}+x^{T} s+w_{0}=\left\|2 x_{0} x_{1}+2 s_{0} s_{1}+x_{0} s_{1}+s_{0} x_{1}+w_{1}\right\| .
$$

By the last relation and (45)-(47), we obtain that (49) holds. To prove (48), we only need to verify $x_{0} v_{1}=v_{0} x_{1}$ and $x_{1}^{T} v_{1}=x_{0} v_{0}$ by the symmetry of $x$ and $s$ in $v$. From (45)-(47) and (49),

$$
\begin{aligned}
x_{0} v_{1} & =x_{0}\left(2 x_{0} x_{1}+2 s_{0} s_{1}+x_{0} s_{1}+s_{0} x_{1}+2 \widetilde{w}_{0} \widetilde{w}_{1}\right) \\
& =2\left(x_{0}^{2}+s_{0}^{2}+x_{0} s_{0}+\widetilde{w}_{0}^{2}\right) x_{1} \\
& =2\left(\left\|x_{1}\right\|^{2}+\left\|s_{1}\right\|^{2}+x_{1}^{T} s_{1}+\frac{w_{0}}{2}\right) x_{1} \\
& =v_{0} x_{1}, \\
x_{1}^{T} v_{1} & =x_{1}^{T}\left(2 x_{0} x_{1}+2 s_{0} s_{1}+x_{0} s_{1}+s_{0} x_{1}+2 \widetilde{w}_{0} \widetilde{w}_{1}\right) \\
& =2 x_{0}\left(\left\|x_{1}\right\|^{2}+s_{0}^{2}+x_{1}^{T} s_{1}+\widetilde{w}_{0}^{2}\right) \\
& =2 x_{0}\left(x_{0}^{2}+s_{0}^{2}+x_{0} s_{0}+\frac{w_{0}}{2}\right) \\
& =x_{0} v_{0} .
\end{aligned}
$$

From (51), the equivalence is also true. 


\section{Jacobian Consistency}

In this section, we will show the Jacobian consistency property and estimate the distance between the gradient of the smoothing function (7) and the subgradient of the WSOCCP complementarity function (5). For any $\mu \in \mathbb{R}, w \in \mathscr{K}^{n}$, let $z:=(x, s, y) \in \mathbb{R}^{n} \times \mathbb{R}^{n} \times \mathbb{R}^{m}$. Based on smoothing function (7), we define $\Phi_{\mu}: \mathbb{R}^{n} \times \mathbb{R}^{n} \times \mathbb{R}^{m} \longrightarrow \mathbb{R}^{2 n+m}$ by

$$
\begin{gathered}
\Phi(z):=\left(\begin{array}{c}
F(x, s, y) \\
\varphi(x, s, w)
\end{array}\right), \\
\Phi_{\mu}(z):=\left(\begin{array}{c}
F(x, s, y) \\
\varphi_{\mu}(x, s, w)
\end{array}\right) .
\end{gathered}
$$

From (1) and (56) and Lemma 1,

$$
\Phi_{\mu}(z)=0 \Leftrightarrow z=(x, s, y) \text { solves WSOCCP (1). }
$$

Since the function $\Phi(z)$ is typically nonsmooth, Newton's method cannot be applied to the system $\Phi(z)=0$ directly. Thus, we can approximately solve the smooth system $\Phi_{\mu}(z)=0$ at each iteration and make $\left\|\Phi_{\mu}(z)\right\|$ decrease gradually by reducing $\mu$ to zero. First, we show that the function $\Phi_{\mu}(z)$ satisfies the Jacobian consistency.

Lemma 5. For any arbitrary but fixed vector $w \in \mathscr{R}^{n}$, we have for any $(\mu, x, s) \in \mathbb{R} \times \mathbb{R}^{n} \times \mathbb{R}^{n}$,

$$
J_{\varphi}^{0}(x, s):=\lim _{\mu \longrightarrow 0} \varphi_{\mu}^{\prime}(x, s, w)=\left(\begin{array}{c}
I-L\left(x+\frac{s}{2}\right) J \\
I-L\left(s+\frac{x}{2}\right) J
\end{array}\right),
$$

where

$$
J:=\left\{\begin{array}{l}
L^{-1}(\omega), \text { if }(x, s) \in \mathscr{I}, \\
\frac{1}{2 \sqrt{2 v_{0}}}\left(1 \bar{v}_{1}^{T} \bar{v} 4 I-3 \bar{v}_{1} \bar{v}_{1}^{T}\right), \text { if }(x, s) \in \mathscr{B}, O, \text { if }(x, s) \in \mathscr{O} .
\end{array}\right.
$$

Proof. By (34) and the symmetry of $x$ and $s$, it suffices to prove

$$
\lim _{\mu \longrightarrow 0} L\left(x+\frac{s}{2}\right) L^{-1}\left(\omega^{\mu}\right)=L\left(x+\frac{s}{2}\right) J
$$

Case 1. If $(x, s) \in \mathscr{I}$, it follows from (25) that

$$
\begin{aligned}
\lim _{\mu \longrightarrow 0} \omega^{\mu} & =\lim _{\mu \longrightarrow 0}\left[\sqrt{\lambda_{1}\left(v^{\mu}\right)} u_{1}(v)+\sqrt{\lambda_{2}\left(v^{\mu}\right)} u_{2}(v)\right] \\
& =\lim _{\mu \longrightarrow 0}\left[\sqrt{\lambda_{1}(v)+\mu^{2}} u_{1}(v)+\sqrt{\lambda_{2}(v)+\mu^{2}} u_{2}(v)\right] \\
& =\sqrt{\lambda_{1}(v)} u_{1}(v)+\sqrt{\lambda_{2}(v)} u_{2}(v) \\
& =\omega \in \operatorname{int} \mathscr{K}^{n} .
\end{aligned}
$$

Therefore,

$$
\lim _{\mu \longrightarrow 0} L\left(x+\frac{s}{2}\right) L^{-1}\left(\omega^{\mu}\right)=L\left(x+\frac{s}{2}\right) L^{-1}(\omega) .
$$

Case 2. If $(x, s) \in \mathscr{B}$, it is easy to prove (51), and

$$
\begin{aligned}
2 v_{0} & =\lambda_{2}(v)>\lambda_{1}(v)=0, \\
\left\|v_{1}\right\| & =v_{0}=\left\|x+\frac{s}{2}\right\|^{2}+\frac{3}{4}\|s\|^{2}+w_{0}>0 .
\end{aligned}
$$

Thus, we obtain the following from (25):

$$
\begin{aligned}
& \lambda_{1}\left(v^{\mu}\right)=\lambda_{1}(v)+\mu^{2}=\mu^{2}>0, \\
& \lambda_{2}\left(v^{\mu}\right)=\lambda_{2}(v)+\mu^{2}=2 v_{0}+\mu^{2}>0 .
\end{aligned}
$$

For any $\mu \neq 0$, we may get from (35) that $L^{-1}\left(\omega^{\mu}\right)=$ $L_{1}\left(v^{\mu}\right)+L_{2}\left(v^{\mu}\right)$. We first prove for any $\mu \neq 0$,

$$
L\left(x+\frac{s}{2}\right) L_{1}\left(v^{\mu}\right)=O
$$

Let

$$
\vartheta:=\left(1 ; \bar{v}_{1}\right)=\frac{1}{\left\|v_{1}\right\|}\left(v_{0} ; v_{1}\right)=\frac{v}{v_{0}} .
$$

Based on (36), (48), and (64), we have

$$
\begin{aligned}
L\left(x+\frac{s}{2}\right) L_{1}\left(v^{\mu}\right) & =\frac{1}{2 \sqrt{\lambda_{1}\left(v^{\mu}\right)}} L\left(x+\frac{s}{2}\right) \vartheta \prime \vartheta^{\prime T} \\
& =\frac{1}{2|\mu|}\left(x+\frac{s}{2}\right) \circ \vartheta \prime \vartheta^{\prime T} \\
& =\frac{1}{2|\mu| v_{0}^{2}}\left(x+\frac{s}{2}\right) \circ v \prime v^{\prime T} \\
& =O .
\end{aligned}
$$

Next, we prove $\lim _{\mu \longrightarrow 0} L_{2}\left(v^{\mu}\right)=J$. From (37), (64), and (65), we have 


$$
\begin{aligned}
\lim _{\mu \longrightarrow 0} L_{2}\left(v^{\mu}\right)= & \lim _{\mu \longrightarrow 0} \frac{1}{2 \sqrt{2 v_{0}+\mu^{2}}}\left(\begin{array}{cc}
1 & \bar{v}_{1}^{T} \\
\bar{v}_{1} & \bar{v}_{1} \bar{v}_{1}^{T}
\end{array}\right) \\
& +\lim _{\mu \longrightarrow 0} \frac{2}{\sqrt{\mu^{2}}+\sqrt{2 v_{0}+\mu^{2}}}\left(\begin{array}{cc}
0 & 0^{T} \\
0 & I-\bar{v}_{1} \bar{v}_{1}^{T}
\end{array}\right) \\
= & \frac{1}{2 \sqrt{2 v_{0}}}\left(\begin{array}{cc}
1 & \bar{v}_{1}^{T} \\
\bar{v}_{1} & 4 I-3 \bar{v}_{1} \bar{v}_{1}^{T}
\end{array}\right)=J .
\end{aligned}
$$

Combining (68) and (69) yields

$\lim _{\mu \longrightarrow 0} L\left(x+\frac{s}{2}\right) L^{-1}\left(\omega^{\mu}\right)=\lim _{\mu \longrightarrow 0} L\left(x+\frac{s}{2}\right) L_{2}\left(v^{\mu}\right)=L\left(x+\frac{s}{2}\right) J$.

Case 3. If $(x, s) \in \mathcal{O}$, it follows from Lemma 4 that $(x, s, w)=(0,0,0)$ and

$$
\begin{aligned}
\omega^{\mu} & =\sqrt{v^{\mu}}=|\mu| e \in \operatorname{int} \mathscr{K}^{n} \\
\lim _{\mu \longrightarrow 0} L\left(x+\frac{s}{2}\right) L^{-1}\left(\omega^{\mu}\right) & =\lim _{\mu \longrightarrow 0} O \cdot \frac{1}{|\mu|} e=O=L\left(x+\frac{s}{2}\right) J .
\end{aligned}
$$

Lemma 6. For any arbitrary but fixed vector $w \in \mathscr{K}^{n}$, we have for any $(x, s) \in \mathbb{R}^{n} \times \mathbb{R}^{n}$,

$$
\left(\begin{array}{c}
I-U_{x} \\
I-U_{s}
\end{array}\right) \in \partial_{B} \varphi(x, s, w),
$$

where

$$
\begin{aligned}
& U_{x}= \pm \frac{1}{2} Z+L\left(x+\frac{s}{2}\right) J, \\
& U_{s}= \pm Z+L\left(s+\frac{x}{2}\right) J, \\
& Z= \begin{cases}O, & \text { if }(x, s) \in \mathscr{I}, \\
\frac{1}{2}\left(1-\bar{v}_{1}^{T}-\overline{v \Psi}_{1} \bar{v}_{1}^{T}\right), & \text { if }(x, s) \in \mathscr{B}, \\
I, & \text { if }(x, s) \in \mathcal{O},\end{cases}
\end{aligned}
$$

and $J$ is defined by (59).

Proof. By Proposition 5.2 [15] and the chain rule for differentiation, the complementarity function $\varphi$ is continuously differentiable at any $(x, s) \in \mathscr{I}$ with

$$
\varphi^{\prime}(x, s, w)=\left(\begin{array}{c}
I-L\left(x+\frac{s}{2}\right) L^{-1}(\omega) \\
I-L\left(s+\frac{x}{2}\right) L^{-1}(\omega)
\end{array}\right) \in \partial_{B} \varphi(x, s, w) .
$$

Thus, it suffices to consider the two cases: $(x, s) \in \mathscr{B}$ and $(x, s) \in \mathcal{O}$.

For any $(x, s) \in \mathscr{B}$ or $(x, s) \in \mathcal{O}$, let $(x, \widehat{s})=(x, s+\mu e)$ with sufficiently small $\mu \neq 0$, and define

$$
\begin{aligned}
& \widehat{v}=\left(\widehat{v}_{0} ; \widehat{v}_{1}\right):=x^{2}+\widehat{s}^{2}+x \circ \widehat{s}+w, \\
& \widehat{\omega}=\left(\widehat{\omega}_{0} ; \widehat{\omega}_{1}\right):=\sqrt{\widehat{v}}, \\
& \widehat{\vartheta}_{1}:=\frac{\widehat{v}_{1}}{\left\|\widehat{v}_{1}\right\|}, \\
& \widehat{\lambda}_{i}=\lambda_{i}(\widehat{v}):=\widehat{v}_{0}+(-1)^{i}\left\|\widehat{v}_{1}\right\|, \quad i=1,2 .
\end{aligned}
$$

Then, we have

$$
\begin{aligned}
\widehat{v} & =x^{2}+(s+\mu e)^{2}+x \circ(s+\mu e)+w \\
& =v+\mu x+2 \mu s+\mu^{2} e, \\
\widehat{v}_{0} & =v_{0}+\mu x_{0}+2 \mu s_{0}+\mu^{2}, \\
\widehat{v}_{1} & =v_{1}+\mu x_{1}+2 \mu s_{1},
\end{aligned}
$$

$\widehat{\lambda}_{i}=v_{0}+\mu x_{0}+2 \mu s_{0}+\mu^{2}+(-1)^{i}\left\|v_{1}+\mu x_{1}+2 \mu s_{1}\right\|, \quad i=1,2$

Obviously, when $\mu \longrightarrow 0$, we have $(x, \widehat{s}) \longrightarrow(x, s)$, $\widehat{v} \longrightarrow v, \widehat{\omega} \longrightarrow \omega$ and $\hat{\lambda}_{i} \longrightarrow \lambda_{i}(v)$ for $i=1,2$. Then by (7), it suffices to show

$$
\begin{aligned}
& \lim _{\mu \longrightarrow 0} L\left(x+\frac{\widehat{s}}{2}\right) L^{-1}(\widehat{\omega})=U_{x}, \\
& \lim _{\mu \longrightarrow 0} L\left(\widehat{s}+\frac{x}{2}\right) L^{-1}(\widehat{\omega})=U_{s},
\end{aligned}
$$

if $\varphi$ is differentiable at $(x, \widehat{s})$.

Case 4. If $(x, s) \in \mathscr{B}$, we obtain $v \in\left(\operatorname{bd} \mathscr{K}^{n} /\{0\}\right)$, and from (45), (46), and (48),

$$
\begin{aligned}
\left\|\widehat{v}_{1}\right\|^{2}= & \left\|v_{1}+\mu x_{1}+2 \mu s_{1}\right\|^{2} \\
= & \left\|v_{1}\right\|^{2}+\mu^{2}\left\|x_{1}\right\|^{2}+4 \mu^{2}\left\|s_{1}\right\|^{2}+4 \mu v_{1}^{T} s_{1} \\
& +2 \mu v_{1}^{T} x_{1}+4 \mu^{2} x_{1}^{T} s_{1} \\
= & \left(v_{0}+\mu x_{0}+2 \mu s_{0}\right)^{2} .
\end{aligned}
$$

The last relation together with $v_{0}>0$ implies that for sufficiently small $\mu$, we have 


$$
\left\|\widehat{v}_{1}\right\|=v_{0}+\mu x_{0}+2 \mu s_{0}>0 .
$$

For sufficiently small $\mu \neq 0$, we obtain from (77) and (80),

$$
\hat{\lambda}_{1}=v_{0}+\mu x_{0}+2 \mu s_{0}+\mu^{2}-\left\|\widehat{v}_{1}\right\|=\mu^{2}>0 \text {, }
$$

$\hat{\lambda}_{2}=v_{0}+\mu x_{0}+2 \mu s_{0}+\mu^{2}+\left\|\widehat{v}_{1}\right\|=2\left(v_{0}+\mu x_{0}+2 \mu s_{0}\right)+\mu^{2}>0$.

It follows from (81) and (82) that $\widehat{v} \in \operatorname{int} \mathscr{K}^{n}$, and hence $\varphi$ is differentiable at $(x, \widehat{s})$.

Now we will prove

$$
\lim _{\mu \longrightarrow 0} L\left(x+\frac{\widehat{s}}{2}\right) L^{-1}(\widehat{\omega})=U_{x}
$$

where $L^{-1}(\widehat{\omega})=L_{1}(\widehat{v})+L_{2}(\widehat{v})$, in which $L_{1}(\widehat{v})$ and $L_{2}(\widehat{v})$ are given by (36) and (37) with $\widehat{v}$ and $\widehat{\vartheta}_{1}$ replacing $v^{\mu}$ and $\bar{v}_{1}$, respectively. By the expression of $\widehat{v}_{1}$ and (80),

$$
\begin{aligned}
\widehat{\vartheta} & :=\left(1 ; \widehat{\vartheta}_{1}\right)=\frac{1}{\left\|\widehat{v}_{1}\right\|}\left(\left\|\widehat{v}_{1}\right\| ; \widehat{v}_{1}\right) \\
& =\frac{1}{\left\|\widehat{v}_{1}\right\|}\left(v_{0}+\mu x_{0}+2 \mu s_{0} ; v_{1}+\mu x_{1}+2 \mu s_{1}\right) \\
& =\frac{1}{\left\|\widehat{v}_{1}\right\|}(v+\mu x+2 \mu s) .
\end{aligned}
$$

By (45), (46), (48), and (84), we have

$$
\begin{aligned}
\left(x+\frac{s}{2}\right) \circ \widehat{\vartheta}_{\prime} & =\frac{1}{\left\|\widehat{v}_{1}\right\|}\left(x+\frac{s}{2}\right) \circ(v+\mu \mathrm{x}+2 \mu \mathrm{s}) \prime \\
& =\frac{1}{\left\|\widehat{v}_{1}\right\|}\left[\left(x+\frac{s}{2}\right) \circ v \prime+2 \mu\left(\mathrm{x}+\frac{\mathrm{s}}{2}\right) \circ\left(\frac{\mathrm{x}}{2}+\mathrm{s}\right) \prime\right] \\
& =0 .
\end{aligned}
$$

Thus, from (36) and (81),

$$
\begin{aligned}
L\left(x+\frac{\widehat{s}}{2}\right) L_{1}(\widehat{v}) & =\frac{1}{2 \sqrt{\widehat{\lambda}_{1}}}\left(x+\frac{s}{2}+\frac{\mu e}{2}\right) \circ \widehat{\vartheta}, \widehat{\vartheta}^{\prime T} \\
& =\frac{1}{2|\mu|}\left[\left(x+\frac{s}{2}\right) \circ \widehat{\vartheta}, \widehat{\vartheta}^{\prime T}+\frac{\mu}{2} \widehat{\vartheta}, \widehat{\vartheta}^{\prime T}\right] \\
& =\frac{\operatorname{sgn}(\mu)}{4} \widehat{\vartheta}_{\prime}, \widehat{\vartheta}^{\prime T} .
\end{aligned}
$$

It follows from (73)-(84) that as $\mu \longrightarrow 0$,

$$
\widehat{\lambda}_{1} \longrightarrow \lambda_{1}(v)=0
$$

$$
\widehat{\lambda}_{2} \longrightarrow \lambda_{2}(v)=2 v_{0} \text {, }
$$$$
\widehat{\vartheta}_{1} \longrightarrow \bar{v}_{1}
$$

$$
\frac{1}{2} \widehat{\vartheta}^{\prime} \widehat{\vartheta}^{\prime T}=\frac{1}{2}\left(\begin{array}{cc}
1 & -\widehat{\vartheta}_{1}^{T} \\
-\widehat{\vartheta}_{1} & --\hat{\vartheta}_{1} \widehat{\vartheta}_{1}^{T}
\end{array}\right) \longrightarrow Z .
$$

Then, by following the proof of Case 5 in Lemma 5, we have

$$
\lim _{\mu \longrightarrow 0} L_{2}(\widehat{v})=\frac{1}{2 \sqrt{2 v_{0}}}\left(\begin{array}{cc}
1 & \bar{v}_{1}^{T} \\
\bar{v}_{1} & 4 I-3 \bar{v}_{1} \bar{v}_{1}^{T}
\end{array}\right)=J .
$$

Therefore, we obtain from (86) and (88) that

$$
\begin{aligned}
\lim _{\mu \longrightarrow \pm 0} L\left(x+\frac{\widehat{s}}{2}\right) L^{-1}(\widehat{\omega})= & \lim _{\mu \longrightarrow \pm 0} L\left(x+\frac{\widehat{s}}{2}\right) L_{1}(\widehat{v}) \\
& +\lim _{\mu \longrightarrow \pm 0} L\left(x+\frac{\widehat{s}}{2}\right) L_{2}(\widehat{v}) \\
= & \lim _{\mu \longrightarrow \pm 0} \frac{\operatorname{sgn}(\mu)}{4} \widehat{\vartheta}^{\prime} \widehat{\vartheta}^{\prime T}+L\left(x+\frac{s}{2}\right) J \\
= & \pm \frac{1}{2} Z+L\left(x+\frac{s}{2}\right) J \\
= & U_{x} .
\end{aligned}
$$

Next we will prove

$$
\lim _{\mu \longrightarrow 0} L\left(\widehat{s}+\frac{x}{2}\right) L^{-1}(\widehat{\omega})=U_{s} .
$$

By (45), (46), (48), (81), and (84), we have

$$
\begin{aligned}
\left(s+\frac{x}{2}\right) \circ \widehat{\vartheta}^{\prime} & =\frac{1}{\left\|\widehat{v}_{1}\right\|}\left(s+\frac{x}{2}\right) \circ(v+\mu \mathrm{x}+2 \mu \mathrm{s}) \prime \\
& =\frac{1}{\left\|\widehat{v}_{1}\right\|}\left[\left(s+\frac{x}{2}\right) \circ v \prime+2 \mu\left(\mathrm{s}+\frac{\mathrm{x}}{2}\right) \circ\left(\frac{\mathrm{x}}{2}+\mathrm{s}\right) \prime\right] \\
& =0,
\end{aligned}
$$

and then 


$$
\begin{aligned}
L\left(\widehat{s}+\frac{x}{2}\right) L_{1}(\widehat{v}) & =\frac{1}{2 \sqrt{\widehat{\lambda}_{1}}}\left(s+\mu e+\frac{x}{2}\right) \circ \widehat{\vartheta}^{\prime} \widehat{\vartheta}^{\prime T} \\
& =\frac{1}{2|\mu|}\left[\left(s+\frac{x}{2}\right) \circ \widehat{\vartheta}, \widehat{\vartheta}^{\prime T}+\mu \widehat{\vartheta}, \widehat{\vartheta}^{\prime T}\right] \\
& =\frac{\operatorname{sgn}(\mu)}{2} \widehat{\vartheta}^{\prime} \widehat{\vartheta}^{\prime T} .
\end{aligned}
$$

Therefore, we obtain from (88) and (92) that

$$
\begin{aligned}
\lim _{\mu \longrightarrow \pm 0} L\left(\widehat{s}+\frac{x}{2}\right) L^{-1}(\widehat{\omega})= & \lim _{\mu \longrightarrow \pm 0} L\left(\widehat{s}+\frac{x}{2}\right) L_{1}(\widehat{v}) \\
& +\lim _{\mu \longrightarrow \pm 0} L\left(\widehat{s}+\frac{x}{2}\right) L_{2}(\widehat{v}) \\
= & \lim _{\mu \longrightarrow \pm 0} \frac{\operatorname{sgn}(\mu)}{2} \widehat{\vartheta}_{\prime}^{\prime} \widehat{\vartheta}^{T}+L\left(s+\frac{x}{2}\right) J \\
= & \pm Z+L\left(s+\frac{x}{2}\right) J \\
= & U_{s} .
\end{aligned}
$$

Case 5. If $(x, s) \in \mathcal{O}$, it follows from Lemma 4 that $(x, s, w)=(0,0,0)$. Thus, $\widehat{v}=\mu^{2} e \in \operatorname{int} \mathscr{K}^{n}, \widehat{\omega}=|\mu| e$, and

$$
\bar{\mu}(x, s, \tau):=\left\{\begin{array}{l}
\frac{\lambda_{1}(v) \tau}{\sqrt{\rho^{2}(x, s)-\lambda_{1}(v) \tau^{2}}} \\
\frac{v_{0} \tau}{\sqrt{2 \rho(x, s)\left(2 \rho(x, s)-\tau \sqrt{2 v_{0}}\right)}} \\
+\infty,
\end{array}\right.
$$

Then, for any $\mu \in \mathbb{R}$ such that $0<|\mu| \leq \bar{\mu}(x, s, \tau)$, we have

$$
\operatorname{dist}\left(\Phi_{\mu_{1}}(z), \partial \Phi(z)\right)<\tau \text {. }
$$

Proof. By (56), it suffices to show the Jacobian consistency of $\varphi_{\mu}$ with $\mu>0$. Define

$$
V^{i}:=\left(\begin{array}{c}
I-U_{x}^{i} \\
I-U_{s}^{i}
\end{array}\right),
$$

$$
\begin{aligned}
\lim _{\mu \longrightarrow \pm 0} L\left(x+\frac{\widehat{s}}{2}\right) L^{-1}(\widehat{\omega}) & =\lim _{\mu \longrightarrow \pm 0} \frac{\mu}{2} I \cdot \frac{1}{|\mu|} I=\lim _{\mu \longrightarrow \pm 0} \frac{\operatorname{sgn}(\mu)}{2} I \\
& = \pm \frac{1}{2} I=U_{x} \\
\lim _{\mu \longrightarrow \pm 0} L\left(\widehat{s}+\frac{x}{2}\right) L^{-1}(\widehat{\omega}) & =\lim _{\mu \longrightarrow \pm 0} \mu I \cdot \frac{1}{|\mu|} I=\lim _{\mu \longrightarrow \pm 0} \operatorname{sgn}(\mu) I \\
& = \pm I=U_{s} .
\end{aligned}
$$

Now we show the Jacobian consistency of the function $\Phi_{\mu}(56)$ and then estimate an upper bound of the parameter $\mu>0$ for the predicted accuracy of the distance between the gradient of $\Phi_{\mu}$ (56) and the subgradient of $\Phi$ (55).

Theorem 1. The following results hold. (i) The function $\Phi_{\mu}$ defined by (56) with $\mu>0$ satisfies the Jacobian consistency. (ii) For given $\tau>0$ and any point $z:=(x, s, y) \in \mathbb{R}^{2 n+m}$, let $\rho(x, s)$ be any function such that

$$
\rho(x, s) \geq\left\|\begin{array}{l}
L\left(x+\frac{s}{2}\right) J \\
L\left(s+\frac{x}{2}\right) J
\end{array}\right\|,
$$

and let $\bar{\mu}: \mathbb{R}^{2 n} \times \mathbb{R}_{+} \longrightarrow \mathbb{R}_{+} \cup\{+\infty\}$ be defined by

$$
\begin{aligned}
& \text { if }(x, s) \in \mathscr{I} \text { and } \tau<\left(\rho(x, s) / \sqrt{\lambda_{1}(v)}\right) \text {, } \\
& \text { if }(x, s) \in \mathscr{B} \text { and } \tau<2 \rho(x, s) / \sqrt{2 v_{0}} \text {, } \\
& \text { otherwise. }
\end{aligned}
$$

where

$$
\begin{aligned}
& U_{x}^{i}=(-1)^{i} \frac{1}{2} Z+L\left(x+\frac{s}{2}\right) J, \\
& U_{s}^{i}=(-1)^{i} Z+L\left(s+\frac{x}{2}\right) J,
\end{aligned}
$$

for $i=1,2, J$ and $Z$ are defined by (59) and (73). Let 


$$
V:=\frac{1}{2}\left(V^{1}+V^{2}\right)=\left(\begin{array}{c}
I-L\left(x+\frac{s}{2}\right) J \\
I-L\left(s+\frac{x}{2}\right) J
\end{array}\right) .
$$

It follows from Lemma 5 and Lemma 6 that

$$
V=J_{\varphi}^{0}(x, s)=\lim _{\mu \longrightarrow 0} \varphi_{\mu_{1}}(x, s, w),
$$

and $V^{1}, V^{2} \in \partial_{B} \varphi(x, s, w)$. Hence,

$$
V=\frac{1}{2}\left(V^{1}+V^{2}\right) \in \partial \varphi(x, s, w),
$$

which together with Definition 1 and Lemma 2 implies the Jacobian consistency of $\varphi_{\mu}$ with $\mu>0$. (ii) For any $z:=(x, s, y) \in \mathbb{R}^{2 n+m}$, it follows from the proof of Theorem 1(i) that

$$
\begin{aligned}
J_{\varphi}^{0}(x, s) & =V \in \partial \varphi(x, s, w), \\
J_{\Phi}^{0}(z) & :=\left(\begin{array}{cc}
J_{\varphi}^{0}(x, s) & O \\
F_{x, s}^{\prime}(x, s, y) & F_{y}^{\prime}(x, s, y)
\end{array}\right) \in \partial \Phi(x, s, y) .
\end{aligned}
$$

Thus, we obtain from (34) and (100) that

$$
\begin{aligned}
\operatorname{dist}\left(\Phi_{\mu}^{\prime}(z), \partial \Phi(z)\right) & \leq\left\|\Phi_{\mu}^{\prime}(z)-J_{\Phi}^{0}(z)\right\| \\
& =\left\|\varphi_{\mu}^{\prime}(z)-J_{\varphi}^{0}(z)\right\| \\
& =\left\|\begin{array}{l}
L\left(x+\frac{s}{2}\right)\left(L^{-1}(\widehat{\omega})-J\right) \| \\
L\left(s+\frac{x}{2}\right)\left(L^{-1}(\widehat{\omega})-J\right)
\end{array}\right\| .
\end{aligned}
$$
have

Then, similar to the proof of Proposition 4.1 [13], we

$$
\operatorname{dist}\left(\Phi_{\mu}^{\prime}(z), \partial \Phi(z)\right) \leq\left|g_{0}(x, s)-g_{\mu}(x, s)\right| \cdot\left\|\begin{array}{c}
L\left(x+\frac{s}{2}\right) J \\
L\left(s+\frac{x}{2}\right) J
\end{array}\right\|
$$

where $g_{\mu}: \mathbb{R}^{2 n} \longrightarrow \mathbb{R}_{+}$is given by

$$
g_{\mu}(x, s):= \begin{cases}\frac{1}{\sqrt{\lambda_{1}(v)+\mu^{2}}}, & \text { if }(x, s) \in \mathscr{I}, \\ \frac{2}{\sqrt{2 v_{0}+\mu^{2}}+|\mu|} \frac{1}{\sqrt{\lambda_{1}(v)+\mu^{2}}}, & \text { if }(x, s) \in \mathscr{B}, \\ 0, & \text { if }(x, s) \in \mathscr{O} .\end{cases}
$$

Hence, by following the proof of Theorem 4.1 [13], the result holds.

\section{Conclusions}

In this paper, we show the Jacobian consistency of the smoothing function $\varphi_{\mu}$ for WSOCCP, which will play a key role in analyzing the rapid convergence of smoothing methods. Moreover, in order to adjust a parameter appropriately in smoothing methods, we estimate the distance between the gradient of the smoothing function $\varphi_{\mu}$ and the subgradient of the weighted SOC complementarity function $\varphi$.

\section{Data Availability}

The data used to support the findings of this study are available from the corresponding author upon request.

\section{Conflicts of Interest}

The authors declare that they have no conflicts of interest.

\section{Acknowledgments}

This research was supported by the National Natural Science Foundation of China (no. 11861026), Guangxi Key Laboratory of Cryptography and Information Security (no. GCIS201819), and Guangxi Key Laboratory of Automatic Detecting Technology and Instruments, China (no. YQ18112).

\section{References}

[1] F. A. Potra, "Weighted complementarity problems---a new paradigm for computing equilibria," SIAM Journal on Optimization, vol. 22, no. 4, pp. 1634-1654, 2012.

[2] K. Anstreicher, "Interior-point algorithms for a generalization of linear programming and weighted centring," Optimization Methods and Software, vol. 27, no. 4-5, pp. 605-612, 2012.

[3] J. Tang, "A variant nonmonotone smoothing algorithm with improved numerical results for large-scale LWCPs," Computational and Applied Mathematics, vol. 37, no. 3, pp. 3927-3936, 2018.

[4] X. Chi, M. S. Gowda, and J. Tao, “The weighted horizontal linear complementarity problem on a Euclidean Jordan algebra," Journal of Global Optimization, vol. 73, no. 1, pp. 153-169, 2019.

[5] S. Pan and J.-S. Chen, "A semismooth Newton method for SOCCPs based on a one-parametric class of SOC complementarity functions," Computational Optimization and Applications, vol. 45, no. 1, pp. 59-88, 2010.

[6] J.-S. Chen and S. Pan, "A one-parametric class of merit functions for the second-order cone complementarity problem," Computational Optimization and Applications, vol. 45, no. 3, pp. 581-606, 2010.

[7] L. Qi, D. Sun, and G. Zhou, "A new look at smoothing Newton methods for nonlinear complementarity problems and box constrained variational inequalities," Mathematical Programming, vol. 87, no. 1, pp. 1-35, 2000.

[8] S. Hayashi, N. Yamashita, and M. Fukushima, "A combined smoothing and regularization method for monotone second- 
order cone complementarity problems," SIAM Journal on Optimization, vol. 15, no. 2, pp. 593-615, 2005.

[9] N. Krejić, S. Rapajić, Globally convergent jacobian smoothing inexact Newton methods for NCP," Computational Optimization and Applications, vol. 41, no. 2, pp. 243-261, 2008.

[10] P. Chen, P. Zhang, X. Zhu X et al., "Modified Jacobian smoothing method for nonsmooth complementarity problems," Computational Optimization and Applications, vol. 75, no. 1, pp. 205-305, 2020.

[11] J. Faraut and A. Korányi, Analysis on Symmetric Cones, Oxford University Press, New York, NY, USA, 1994.

[12] X. Chen, L. Qi, and D. Sun, "Global and superlinear convergence of the smoothing Newton method and its application to general box constrained variational inequalities," Mathematics of Computation, vol. 67, no. 222, pp. 519-541, 1998.

[13] H. Ogasawara and Y. Narushima, "The Jacobian consistency of a smoothed Fischer-Burmeister function associated with second-order cones," Journal of Mathematical Analysis and Applications, vol. 394, no. 1, pp. 231-247, 2012.

[14] F. Clarke, Optimization and Nonsmooth Analysis, John Wiley \& Sons, New York, NY, USA, 1983.

[15] M. Fukushima, Z. Luo, and P. Tseng, "Smoothing functions for second-order-cone complementarity problems," SIAM Journal on Optimization, vol. 12, no. 2, pp. 436-460, 2001.

[16] J. Chen and P. Tseng, "An unconstrained smooth minimization reformulation of the second-order cone complementarity problem," Mathematical ProgrammingD, vol. 104, no. 2-3, pp. 293-327, 2005. 